\title{
Leitura em Voz Alta e Vizinhança Ortográfica: Acesso Lexical e Processamento Estratégico
}

\author{
Reading Aloud Tasks and Orthographic Neighborhood: \\ Lexical Access and Strategic Processing
}

\author{
Francis Ricardo dos Reis Justi*,a, Antonio Roazzi ${ }^{b}$ \& Cláudia Nascimento Guaraldo Justi ${ }^{a}$ \\ ${ }^{a}$ Universidade Federal de Juiz de Fora, Juiz de Fora, Minas Gerais, Brasil \\ $\&{ }^{b}$ Universidade Federal de Pernambuco, Recife, Pernambuco, Brasil
}

\begin{abstract}
Resumo
Dois experimentos foram realizados visando investigar o efeito do número de vizinhos ortográficos (N) e do número de vizinhos ortográficos de maior frequência de ocorrência (NF) em tarefas de leitura em voz alta (TLVA). Os estímulos experimentais consistiram em 64 palavras correspondentes a uma manipulação fatorial $2 \times 2$ de N e NF. No primeiro experimento, uma TLVA do tipo Go/NoGo, observou-se um efeito inibidor de NF quando as palavras tinham muitos vizinhos ortográficos. No segundo experimento, uma TLVA na qual tanto as palavras quantos as pseudopalavras deviam ser pronunciadas em uma mesma sessão, observou-se um efeito facilitador de N. Os efeitos de N e NF foram atribuídos a diferenças de ênfase entre as tarefas no processo de acesso lexical.

Palavras-chave: Reconhecimento visual de palavras, vizinhança ortográfica, acesso lexical, leitura.

Abstract

Two experiments were carried out to investigate the orthographic neighborhood density $(\mathrm{N})$ and neighborhood frequency effects (NF) in reading aloud tasks. The experimental stimuli consisted of 64 words corresponding to a $2 \times 2$ factorial manipulation of N and NF. In the first experiment, during a Go/NoGo reading aloud task, an inhibitory NF effect was observed when the words had a high number of neighbors. In the second experiment, a reading aloud task in which the subjects had to pronounce words and pseudowords mixed in the same session, a facilitative $\mathrm{N}$ effect was observed. $\mathrm{N}$ and NF effects were attributed to different task demands on the process of lexical access.

Keywords: Visual word recognition, orthographic neighborhood, lexical access, reading.
\end{abstract}

É comum modelos de reconhecimento visual de palavras postularem a existência de um "dicionário mental" onde o leitor armazena as representações lexicais das formas ortográficas das palavras que são lidas (Coltheart, Rastle, Perry, Langdon, \& Ziegler, 2001; Grainger \& Jacobs, 1996; McClleland, \& Rumelhart, 1981). Assim sendo, uma das principais tarefas que o nosso sistema cognitivo desempenha durante o acesso a essas representações (acesso lexical) é a seleção da entrada lexical correta diante

\footnotetext{
* Endereço para correspondência: Universidade Federal de Juiz de Fora, ICH, Departamento de Psicologia, Rua José Lourenço Kelmer, s/n, Campus Universitário, Bairro São Pedro, CEP 36036-900, Juiz de Fora, MG, Brasil. E-mail: francisjusti@gmail.com.

Esse estudo é parte integrante da tese de doutorado do primeiro autor. Assim sendo, os participantes desse estudo participaram também de outros experimentos, porém, esses foram desenvolvidos com estímulos e tarefas diferentes e, por questões de espaço, serão apresentados em outros trabalhos.

Agradecimentos: Os autores agradecem, também, ao Conselho Nacional de Desenvolvimento Científico e Tecnológico $(\mathrm{CNPq})$ por financiar suas atividades.
}

das diversas entradas que podem ser ortograficamente similares ao estímulo alvo. $\mathrm{O}$ efeito que a similaridade ortográfica de uma palavra exerce no processo de acesso lexical é conhecido por efeito de vizinhança ortográfica e tem sido questão bastante estudada na literatura sobre o reconhecimento visual de palavras (Adelman \& Brown, 2007; Andrews, 1989, 1997; Carreiras, Perea, \& Grainger, 1997; Coltheart, Davelaar, Jonasson, \& Besner, 1977; Grainger, O'Regan, Jacobs, \& Segui, 1989; Huntsman \& Lima, 2002; Justi \& Pinheiro, 2006, 2008; Mulatti, Reynolds, \& Besner, 2006; Peereman \& Content, 1995; Perea \& Pollatsek, 1998; Perea \& Rosa, 2000; Perry, Lupker, \& Davis, 2008; Siakaluk, Sears, \& Lupker, 2002).

As medidas de vizinhança ortográfica mais utilizadas são as medidas N (de Neighborhood Density) e NF (de Neighborhood Frequency). A medida $\mathrm{N}$ foi proposta por Coltheart et al. (1977) para indexar o número de palavras que podem ser criadas a partir de uma palavra alvo mudando-se uma de suas letras, enquanto a posição das demais é preservada. Assim sendo, considera-se que a palavra 'alma' tenha cinco vizinhos ortográficos $(\mathrm{N}=5)$, pois a partir da mudança de uma de suas letras podem ser 
geradas as palavras 'arma', 'asma', 'alga', 'alça' e 'alta'. A medida NF foi proposta por Grainger et al. (1989) e refere-se à frequência de ocorrência dos vizinhos ortográficos da palavra alvo, ou seja, se ela possui vizinhos de frequência maior do que a dela ou não (p.ex., a palavra 'capa' tem um vizinho ortográfico mais frequente que é a palavra 'casa').

De acordo com Perea e Rosa (2000), os estudos sobre os efeitos de $\mathrm{N}$ e NF no acesso lexical são importantes porque podem ajudar a revelar a dinâmica do processo de acesso às representações lexicais postuladas pelos modelos de reconhecimento visual de palavras (Coltheart et al., 2001; Grainger \& Jacobs, 1996; McClleland, \& Rumelhart, 1981). Por exemplo, esse processo implicaria em uma espécie de "competição" entre as unidades lexicais que são similares ao estímulo alvo? Se este for o caso, o esperado é que os efeitos de $\mathrm{N}$ e NF sejam inibidores, ou seja, esperar-se-ia um decréscimo na rapidez ou precisão no reconhecimento de palavras em função do aumento do número de vizinhos ortográficos $(\mathrm{N})$ e/ou da presença de um vizinho ortográfico de maior frequência (NF). Um efeito facilitador de $\mathrm{N}$ e NF implicaria no padrão oposto e seria uma evidência contra a ideia de um mecanismo de competição em nível lexical. Dada a sua importância teórica, diversos estudos sobre o efeito de vizinhança ortográfica têm sido realizados com falantes de diferentes línguas, porém os resultados têm sido um tanto conflitantes, com os efeitos de $\mathrm{N}$ e NF parecendo variar de nulos a facilitadores ou inibidores, de acordo com o tipo de tarefa utilizada e a língua na qual foi realizada a pesquisa (para uma revisão da literatura ver os trabalhos de Andrews, 1997; Mathey, 2001 e Perea \& Rosa, 2000).

Uma hipótese explicativa para esses achados conflitantes é a de Andrews (1997) que procura enfatizar diferenças existentes entre a língua inglesa e as demais línguas nas quais as pesquisas foram realizadas. De acordo com Andrews, na língua inglesa, as palavras monossílabas que têm muitos vizinhos ortográficos, têm uma maior probabilidade de também terem vizinhos que compartilham a mesma rima (body). A relação entre essas variáveis é importante, pois alguns trabalhos têm demonstrado que palavras que têm vizinhos que compartilham a mesma rima (body neighbors) são reconhecidas mais rapidamente na língua inglesa (Treiman, Mullennix, Bijeljac-Babic, \& Richmond-Welty, 1995; Ziegler \& Perry, 1998). Isso aconteceria porque, no inglês, a pronúncia da vogal nos monossílabos é bastante inconsistente e a rima, nessas palavras, ajudaria a prever a pronúncia dessa vogal (Treiman et al., 1995). No entanto, essa proposta de Andrews (1997) enfrenta dificuldades. Um dos problemas com a proposta é que ela não explica porque as diferenças entre as línguas não se manifestam em todas as tarefas. Estudos dos movimentos dos olhos na leitura têm revelado efeitos inibidores de NF, tanto na língua inglesa (Perea \& Pollatsek, 1998) quanto na francesa (Grainger et al., 1989). Além disso, se a diferença nos resultados encontrados nas tarefas deve-se, principalmente, ao fato de que, na língua inglesa, as palavras com muitos vizinhos ortográficos teriam uma pronúncia mais previsível, era de se esperar uma diferença ainda maior entre as línguas na tarefa de leitura em voz alta, já que nessa tarefa a pronúncia dos estímulos é obrigatória. No entanto, isso não ocorre, sendo observado um efeito facilitador de $\mathrm{N}$ em falantes de diferentes línguas na tarefa de leitura em voz alta (p.ex., Andrews, 1989 no inglês; Carreiras et al., 1997 no espanhol; Peereman \& Content, 1995 no francês).

Uma explicação alternativa para os resultados conflitantes que têm sido relatados é argumentar que o efeito de vizinhança ortográfica ocorre em dois momentos e de maneiras diferentes (Perea \& Rosa, 2000). Em um primeiro momento, no qual o importante seria decodificar o estímulo visual em um conjunto de letras, o efeito de vizinhança ortográfica seria facilitador (em especial, o efeito de $\mathrm{N}$ ). Já em um segundo momento, quando o importante seria selecionar a representação lexical correspondente ao estímulo alvo, o efeito seria inibidor (nesse caso, o efeito de NF seria preponderante). Assim sendo, em tarefas onde a ênfase não é no acesso ao léxico, o efeito facilitador do número de vizinhos ortográficos tornar-se-ia mais evidente. Já em tarefas nas quais o acesso ao léxico está mais envolvido, o efeito inibidor de NF seria preponderante. A dificuldade com essa proposta é que não parece existir uma tarefa "pura" que avalie, exclusivamente, o processo de acesso lexical (Andrews, 1997). Geralmente, qualquer tarefa utilizada envolve aspectos relacionados ao processo de acesso lexical, mas também envolve demandas a outros processos necessários ao desempenho na tarefa (Monsell, Doyle, \& Haggard, 1989).

Uma forma de se lidar com a questão do grau em que o acesso lexical ocorre em diferentes tarefas é desenvolver um modelo que especifique os possíveis componentes envolvidos nas tarefas em questão. Grainger e Jacobs (1996) desenvolveram um modelo desse tipo relacionado à tarefa de decisão lexical e o utilizaram para demonstrar que os efeitos de $\mathrm{N}$ e NF podem variar de acordo com a ênfase que é colocada no processo de acesso lexical. De acordo com esses autores, como na tarefa de decisão lexical o participante deve decidir se um estímulo é uma palavra ou uma pseudopalavra ${ }^{1}$, sua resposta pode ser afetada pelo grau em que as pseudopalavras são ortograficamente similares a palavras. Assim sendo, quanto mais ortograficamente similares a palavras forem as pseudopalavras, mais difícil será discriminar os dois tipos de estímulo e os participantes terão de finalizar o processo de acesso lexical para decidir se o estímulo alvo é uma palavra ou não. Já quando as pseudopalavras forem pouco similares a palavras, os participantes podem responder com base na familiaridade inicial do estímulo sem, necessariamente, completar o processo de acesso lexical. Com base nesse raciocínio, Grainger e Jacobs (1996) desenvolveram duas

\footnotetext{
${ }^{1}$ Sequência de letras construída com estrutura ortográfica possível na língua em questão, mas não associada a nenhum significado. Por exemplo, no caso do português brasileiro, 'bapana' é uma pseudopalavra.
} 
variações da tarefa de decisão lexical para investigar os efeitos de N e NF em função das pseudopalavras utilizadas no estudo. No primeiro experimento, pseudopalavras com muitos vizinhos ortográficos foram utilizadas e encontrou-se um efeito nulo de $\mathrm{N}$ e um efeito inibidor de NF. No segundo experimento, pseudopalavras com poucos vizinhos ortográficos foram utilizadas e encontrou-se um efeito facilitador de N, no entanto, o efeito inibidor de NF permaneceu.

Desde então, com base no trabalho de Grainger e Jacobs (1996), pesquisadores têm comparado os efeitos de $\mathrm{N}$ e NF em versões diferentes da tarefa de decisão lexical. Por exemplo, em um estudo com falantes do espanhol, Carreiras et al. (1997) observaram que o efeito de $\mathrm{N}$ variou de nulo a facilitador de acordo com a facilidade ou dificuldade de se discriminar as palavras das pseudopalavras na tarefa de decisão lexical, enquanto o efeito de NF foi inibidor em ambas as tarefas. Em um estudo realizado com estudantes universitários falantes do português brasileiro, Justi e Pinheiro (2006) investigaram o efeito de $\mathrm{N}$ e $\mathrm{NF}$ em duas versões da tarefa de decisão lexical. A primeira delas foi uma tarefa de decisão lexical do tipo Go/No-Go, na qual são apresentadas em ordem variada palavras e pseudopalavras, mas só se requer que o participante responda às palavras. Nessa tarefa, foram utilizadas palavras e pseudopalavras que tinham um número de vizinhos ortográficos equiparável e observou-se um efeito inibidor de NF quando as palavras tinham poucos vizinhos e um efeito inibidor de $\mathrm{N}$ quando as palavras não tinham vizinhos de maior frequência. Já na segunda tarefa de decisão lexical, as pseudopalavras não tinham vizinhos ortográficos e o tempo de apresentação dos estímulos era limitado, nesse caso, observou-se um efeito facilitador de N. Em um estudo realizado com uma amostra de crianças do quinto ano do ensino fundamental, Justi e Pinheiro (2008) utilizaram as mesmas versões da tarefa de decisão lexical de seu estudo anterior. Na tarefa de decisão lexical do tipo Go/NoGo, efeitos inibidores de N e NF foram observados e na tarefa de decisão lexical com tempo fixo, observou-se um efeito inibidor de NF e um efeito nulo de N. Dessa forma, Justi e Pinheiro (2008) postularam que a tarefa de decisão lexical do tipo Go/NoGo avalia mais fidedignamente o acesso lexical genuíno, posto que nessa, o padrão de respostas de adultos (Justi \& Pinheiro, 2006) e crianças (Justi \& Pinheiro, 2008) foi semelhante. Por fim, outro estudo que também investigou a variação no efeito de $\mathrm{N}$ e NF em função da ênfase dada ao acesso lexical na tarefa de decisão lexical foi o de Siakaluk et al. (2002). Porém, nesse estudo, não foram observados efeitos inibidores de $\mathrm{N}$ ou NF, mesmo quando as pseudopalavras tinham muitos vizinhos ortográficos. No entanto, uma falha metodológica no estudo de Siakaluk et al. (2002) é que os autores não controlaram o número de body neighbors e como o estudo foi realizado com falantes da língua inglesa, isso pode ter afetado os resultados do estudo, já que essa variável tem um efeito facilitador na língua inglesa (Treiman et al., 1995;
Ziegler \& Perry, 1998) e se correlaciona com o número de vizinhos ortográficos (Andrews, 1997).

Embora os efeitos de N e NF pareçam, de fato, variar à medida que a ênfase no acesso lexical aumenta ou diminui, é importante considerar que os estudos de Carreiras et al. (1997), Grainger e Jacobs (1996) e Justi e Pinheiro (2006, 2008) se restringiram a avaliar a hipótese desses efeitos serem modulados apenas na tarefa de decisão lexical. No entanto, a proposta de Perea e Rosa (2000) versa sobre os efeitos de N e NF no processo de acesso lexical de forma mais geral e o fato dessa variação ter sido explicitamente investigada apenas na tarefa de decisão lexical representa uma clara lacuna na literatura da área. Afinal, se os efeitos de N e NF podem variar conforme a ênfase que é colocada no processo de acesso lexical, então é importante investigar se em outras tarefas essa ênfase no processo de acesso lexical também pode ser variada. Caso isso seja possível, poder-se-ia melhor argumentar a favor da generalidade da proposta de Perea e Rosa (2000). Assim sendo, o presente estudo objetivou ajudar a suprir essa lacuna na literatura, investigando se os efeitos de N e NF também podem ser modulados por variações na ênfase no processo de acesso lexical em duas versões da tarefa de leitura em voz alta.

$\mathrm{O}$ presente estudo procurou atender a esse objetivo a partir da realização de dois experimentos. No primeiro experimento, utilizou-se uma tarefa de leitura em voz alta do tipo Go/NoGo tendo como objetivo enfatizar o processo de acesso lexical. Em uma tarefa de leitura em voz alta do tipo Go/NoGo o participante é exposto à palavras e pseudopalavras em ordem aleatória, porém é solicitado a ler em voz alta apenas as palavras. Assim sendo, o desempenho bem sucedido nessa tarefa demanda que o participante identifique lexicalmente o estímulo antes de pronunciá-lo, enfatizando, assim, o processo de acesso lexical (Justi \& Pinheiro, 2006, 2008). Dessa forma, se os argumentos de Perea e Rosa (2000) estiverem corretos, deve-se observar na tarefa de leitura em voz alta do tipo Go/NoGo: (a) um efeito inibidor de $\mathrm{N}$ e NF e (b) que o efeito de NF deve ser mais forte do que o efeito de $\mathrm{N}$.

No segundo experimento desenvolvido, utilizou-se uma tarefa de leitura na qual os participantes deviam ler em voz alta palavras e pseudopalavras que apareciam em ordem aleatória em uma mesma sessão. De acordo com Coltheart (1978), se em uma tarefa de leitura em voz alta são misturadas palavras e pseudopalavras e se requer dos participantes respostas a ambos os estímulos, é possível que esses tendam a ler "estrategicamente" todos os estímulos utilizando um processo de conversão grafema/ fonema, sem, necessariamente, acessar a representação lexical específica da palavra alvo. Dessa forma, como a ênfase nessa tarefa não é no acesso ao léxico, mas na conversão de um conjunto de grafemas em um conjunto de fonemas, se os argumentos de Perea e Rosa (2000) estiverem corretos, deve-se observar nessa tarefa: (a) um efeito facilitador de $\mathrm{N}$ e NF e (b) que o efeito de $\mathrm{N}$ deve ser mais forte que o de NF. 


\section{Método}

\section{Participantes}

Participaram dos dois experimentos 32 estudantes de Psicologia falantes do português brasileiro. A média de idade dos participantes foi de 21 anos e 2 meses, sendo 24 do sexo feminino e 8 do sexo masculino. Esse estudo foi aprovado pelo Comitê de Ética em Pesquisa da universidade na qual foi realizado (número de registro 018238/2008-06).

\section{Experimento A - Tarefa de Leitura em Voz Alta do Tipo Go/NoGo}

O objetivo desse experimento é observar os efeitos de $\mathrm{N}$ e NF quando o processo de acesso lexical é enfatizado. Para tal, utilizou-se a tarefa de leitura em voz alta do tipo Go/NoGo, já que essa demanda a identificação lexical do estímulo antes de sua pronúncia.

\section{Material}

Os estímulos experimentais consistiram de 64 palavras que atenderam a uma manipulação fatorial 2 x 2 de N (um vizinho ortográfico ou quatro ou mais vizinhos ortográficos) e NF (sem vizinhos ortográficos de maior frequência de ocorrência ou com um vizinho ortográfico de maior frequência). Essas 64 palavras foram retiradas do trabalho de Justi e Justi (2008) que traz estatísticas de vizinhança ortográfica para palavras do português brasileiro. Além dessas 64 palavras atenderem a uma manipulação fatorial de $\mathrm{N}$ e $\mathrm{NF}$, as seguintes características dessas foram controladas: a frequência de ocorrência (foram utilizadas palavras de baixa frequência de ocorrência, tendo essas, em média, 10,11 ocorrências por milhão de palavras); o número de sílabas (todas dissílabas); o grau de correspondência no mapeamento grafema-fonema (todas regulares); a classe gramatical (apenas substantivos); e, a natureza do fonema inicial (as palavras foram pareadas entre as condições experimentais de acordo com o seu fonema inicial). No caso do pareamento das palavras de acordo com o seu fonema inicial, a única exceção configurou-se no pareamento do fonema inicial /õ/ da palavra 'onze' com o fonema inicial /a/ das palavras 'astro', 'asas' e 'Ásia'. No entanto, Pinheiro e Reis (2004) não observaram diferenças estatisticamente significativas entre as médias de tempo de reação das palavras iniciadas com esses fonemas (/õ/ e /a/) e por isso esse pareamento foi mantido. A lista de palavras utilizadas nesse estudo encontra-se no Anexo.

Por fim, para compor a tarefa de leitura em voz alta do tipo Go/NoGo, foram selecionadas 16 pseudopalavras dissílabas com muitos vizinhos ortográficos (média de 5,88 e desvio-padrão de 2,99 vizinhos ortográficos). Foram selecionadas pseudopalavras com muitos vizinhos ortográficos porque, de acordo com Grainger e Jacobs (1996), essas geram mais ativação lexical, reduzindo assim, a chance do participante distinguir entre as palavras e as pseudopalavras do estudo com base apenas em sua familiaridade.

\section{Procedimentos}

As 64 palavras correspondentes à manipulação fatorial de N e NF foram subdivididas em quatro listas (pareadas pela natureza do fonema inicial e balanceadas pela frequência de ocorrência média) que foram cruzadas entre as quatro condições experimentais. A ordem de apresentação dessas listas foi contrabalanceada entre as quatro condições experimentais, de forma que cada participante foi exposto a apenas uma lista por condição experimental, configurando, assim, um total de 16 palavras $^{2}$. Assim sendo, na sessão experimental, cada participante foi exposto a 32 estímulos, sendo 16 palavras e 16 pseudopalavras. Esse experimento contou, também, com uma sessão de treinamento composta de oito estímulos adicionais, sendo quatro palavras e quatro pseudopalavras com características semelhantes às das palavras e pseudopalavras utilizadas na sessão experimental.

Os participantes foram testados individualmente em uma sala cedida pela instituição, onde foram recebidos e instruídos por um colaborador do pesquisador quanto aos procedimentos. É importante acrescentar que o colaborador não tinha conhecimento das hipóteses experimentais, sendo esse um experimento do tipo duplo-cego. O instrumento utilizado para o teste foi um computador de arquitetura compatível à IBM-PC e o software utilizado para a apresentação dos estímulos e coleta dos dados de tempo de reação (TR) e porcentagem de erros foi o DMDX (Forster $\&$ Forster, 2003) $)^{3}$. O participante se acomodava na cadeira, ficando a uma distância aproximada de 40 centímetros da tela do computador, dependendo de sua postura. Após se acomodar, o colaborador pedia ao participante para falar algumas palavras com a finalidade de calibrar o microfone e então o instruía a ler as instruções experimentais na tela do computador. As instruções foram as seguintes:

Nesse teste você verá esse sinal + no centro da tela. Logo após, você verá uma palavra real ou uma palavra inventada. Fale o nome do item apenas quando for

2 O esquema de contrabalanceamento utilizado foi o mesmo proposto por Pollatsek e Well (1995) no anexo 'B' de seu trabalho. Esse contrabalanceamento de listas permite o cálculo da variância referente ao efeito principal das listas e da variância referente à interação entre listas e tratamento. Isso permitiu que na análise estatística realizada nesse estudo a variância referente à interação entre listas e tratamento fosse acrescentada ao termo de erro do teste estatístico, evitando dessa forma o problema conhecido como a "falácia da linguagem como um efeito fixo" (Raaijmakers, Schrijnemakers, \& Gremmen, 1999). Veja-se o anexo B do trabalho de Pollatsek e Well (1995) para uma discussão detalhada do design utilizado nesse estudo. Os trabalhos de Clark (1973) e Raaijmakers et al. (1999) discutem o problema da "falácia da linguagem como um efeito fixo". Outra vantagem desse contrabalanceamento de listas é que como cada participante foi exposto a apenas $1 / 4$ das palavras foi possível utilizar a mesma lista de palavras nos dois experimentos sem que um participante fosse exposto à mesma palavra duas vezes.

$3 \quad$ O TR foi mensurado pelo tempo decorrido entre o aparecimento do estímulo e o início da resposta vocal de um participante e os estímulos alvo foram apresentados em ordem aleatória para cada participante. O software DMDX (Forster \& Forster, 2003) tem uma precisão de milissegundos na dinâmica de apresentação dos estímulos e registro do TR. 
uma palavra real, do contrário espere ele desaparecer. Tente fazer isso o mais rápido possível, mas evite errar. Aperte o botão 3 para iniciar o treinamento.

Após ler essas instruções o participante tinha a oportunidade de redimir quaisquer dúvidas antes de iniciar a sessão de treinamento ou logo após essa, porém, antes de iniciar a sessão experimental.

Os estímulos foram apresentados em uma configuração de tela de 640x480 pixels, na fonte 'fixedsys' de tamanho 10 e em letras capitulares, sendo a cor da fonte branca e o fundo azul. Na sessão experimental, as palavras e pseudopalavras tiveram a seguinte dinâmica de apresentação: a apresentação de cada estímulo alvo (palavra ou pseudopalavra) iniciava-se com uma marca de fixação $(+)$ que aparecia na tela por 500 milissegundos (ms) e depois desaparecia deixando a tela sem estímulos por $500 \mathrm{~ms}$; aparecia então o estímulo alvo e esse permanecia na tela por $1500 \mathrm{~ms}$ ou até que a pessoa emitisse uma resposta vocal. $\mathrm{O}$ intervalo entre o fim da apresentação de um estímulo alvo e o início da apresentação de outro foi de $500 \mathrm{~ms}$. O participante devia ler em voz alta o estímulo alvo apenas quando esse fosse uma palavra, do contrário deveria esperar que desaparecesse. A sessão de treinamento seguiu a mesma dinâmica de apresentação da sessão experimental, porém contou com feedback quanto à precisão das respostas dadas. Caso o participante apresentasse uma porcentagem de erros maior do que $15 \%$ na sessão de treinamento, essa era automaticamente repetida. O tempo total de testagem, incluindo as sessões de treinamento e experimental, foi de, aproximadamente, 5 minutos por participante.

\section{Resultados $^{4}$}

Optou-se nesse estudo por utilizar o método das medidas semirrestringidas (Perea, 1999) para lidar com a distribuição positivamente assimétrica do TR e, por conseguinte, os escores de um participante que se desviaram de sua média, por mais de dois desvios padrão em qualquer direção, foram limitados a esse valor. Como as pseudopalavras não deviam ser pronunciadas, esse procedimento foi aplicado apenas aos escores de TR para as palavras e alterou $4,3 \%$ desses escores resultando em uma distribuição normal do TR (Kolmogorov-Smirnov $Z=$ $1,016, p>0,25)$. Ao responder às palavras, os participantes apresentaram um TR médio de $835,49 \mathrm{~ms}$, com um desvio padrão de $154,92 \mathrm{~ms}$. A Tabela 1 apresenta as estatísticas descritivas do TR médio dos participantes por condição experimental.

Tabela 1

Média e Desvio Padrão do TR na Tarefa de Leitura em Voz Alta do Tipo Go/NoGo

\begin{tabular}{|c|c|c|c|c|}
\hline & \multicolumn{2}{|c|}{ N1 } & \multicolumn{2}{|c|}{$\mathrm{N} 2$} \\
\hline & NF1 & NF2 & NF1 & NF2 \\
\hline Média & 833,78 & 832,59 & 810,84 & 867,22 \\
\hline Desvio Padrão & 168,10 & 198,11 & 159,35 & 165,65 \\
\hline
\end{tabular}

Nota. N1 = apenas 1 vizinho ortográfico; $\mathrm{N} 2=4$ ou mais vizinhos ortográficos; $\mathrm{NF} 1=$ sem vizinhos ortográficos de maior frequência de ocorrência; NF2 = um vizinho ortográfico de maior frequência de ocorrência.

Uma análise de variância considerando N e NF como fatores intra-sujeitos com dois níveis cada e considerando o esquema de contrabalanceamento de listas a partir de dois fatores entre-sujeitos foi desenvolvida de acordo com as recomendações de Pollatsek e Well (1995, anexo B) tendo como variável dependente o TR (vide nota 2 para mais informações sobre o design e análise estatística utilizados no presente estudo). Como os fatores entre sujeitos foram incluídos apenas por permitir acrescentar ao termo de erro do teste estatístico a variância referente à interação entre listas e tratamento, os efeitos desses fatores não foram relatados abaixo para facilitar a visualização dos resultados.

Outro fator relevante é que como a partir da proposta de Perea e Rosa (2000) podem ser feitas predições direcionais claras para o efeito de vizinhança ortográfica (no caso dessa tarefa, é predito um efeito inibidor), optou-se por fazer uso de análises unicaudais para avaliar os efeitos principais de N e NF nesse estudo por serem análises mais poderosas do ponto de vista estatístico (as outras análises foram sempre bicaudais). Assim sendo, considerando-se o efeito principal de $\mathrm{N}$, as palavras com quatro ou mais vizinhos ortográficos foram reconhecidas ligeiramente mais devagar (média de $839,03 \mathrm{~ms}$ ) do que as palavras com apenas um vizinho ortográfico (média de $833,19 \mathrm{~ms}$ ). No entanto, essa diferença não foi estatisticamente significativa $[F(1,30)=0,282 ; p=0,3$ unicaudal $]$. As palavras com um vizinho ortográfico de maior frequência de ocorrência também foram reconhecidas mais lentamente (média de $849,91 \mathrm{~ms}$ ) do que as palavras sem vizinhos de maior frequência de ocorrência (média de $822,31 \mathrm{~ms}$ ), porém, o efeito principal de NF também não foi estatisticamente significante $[F(1,30)=1,896 ; p=0,09$ unicaudal $]$. Já a interação entre $\mathrm{N}$ e NF foi estatisticamente significante $[F$ $(1,30)=4,152 ; p=0,05]$. 
Para explorar a interação observada entre N e NF quatro testes post hoc foram desenvolvidos para comparar as seguintes médias da Tabela 1: NF1 x NF2 quando as palavras tinham apenas um vizinho ortográfico (N1); NF1 $\mathrm{x}$ NF2 quando as palavras tinham quatro ou mais vizinhos ortográficos (N2); N1 x N2 quando as palavras não tinham vizinhos ortográficos de maior frequência de ocorrência (NF1); e, N1 x N2 quando as palavras tinham um vizinho ortográfico de maior frequência de ocorrência (NF2). Para as comparações tipo post hoc optou-se por seguir as recomendações de Warner (2008) e computar testes $t$ para medidas repetidas e ajustar o nível de significância de acordo com o método de Bonferroni. Assim sendo, como nas análises post hoc foram realizadas quatro comparações, só foram consideradas estatisticamente significativas (em um nível $\alpha$ de 0,05 ) as comparações cujos valores de $p$ foram menores do que 0,0125 . Os resultados das análises post hoc indicaram que a interação ocorreu porque quando as palavras tinham muitos vizinhos ortográficos (N2), a presença de um vizinho ortográfico de maior frequência de ocorrência aumentou a média de TR dos participantes em, aproximadamente, $56 \mathrm{~ms}$ [NF1 x NF2: $t(31)=2,750$; $p=0,01]$, ou seja, o efeito inibidor de NF se restringiu às palavras que tinham muitos vizinhos ortográficos. Nenhum dos outros testes post hoc foram significantes (para todos $p>0,11)$. Por fim, conforme o esperado por se tratarem de leitores hábeis, a média de porcentagem de erros foi ínfima $(1,95 \%)$ e os dados referentes a essa medida não foram analisados.

\section{Discussão}

Em certo sentido, pode-se dizer que os resultados foram consistentes com as hipóteses experimentais, já que os efeitos de $\mathrm{N}$ e NF apresentaram a direção prevista e o efeito inibidor de NF (aproximadamente, $28 \mathrm{~ms}$ ) foi numericamente maior do que o efeito inibidor de $\mathrm{N}$ (aproximadamente, 6ms). No entanto, é importante ressaltar que os efeitos principais dessas variáveis não foram significantes. Isso ocorreu devido a uma interação entre N e NF, onde se pôde observar que a presença de um vizinho ortográfico de maior frequência de ocorrência somente retardou o reconhecimento da palavra alvo quando essa tinha muitos vizinhos ortográficos. Assim sendo, uma explicação adequada dos efeitos de $\mathrm{N}$ e NF nessa tarefa deve ser capaz de acomodar a interação entre essas variáveis.

De acordo com Perea e Rosa (2000), efeitos inibidores de $\mathrm{N}$ e NF estariam relacionados ao processo de acesso lexical. Isso aconteceria porque, para esses autores, o processo de acesso lexical implicaria em uma espécie de "competição" entre as unidades lexicais que são similares ao estímulo alvo como previsto pelos modelos de Coltheart et al. (2001), Grainger e Jacobs (1996) e McClleland e Rumelhart (1981). Desse modo, palavras que tivessem muitos vizinhos ortográficos ou vizinhos ortográficos mais frequentes teriam ou mais competidores ou competidores "mais fortes" e demorariam mais para serem reconhecidas.
A questão então passa pela possibilidade de se compatibilizar a ideia de um mecanismo de competição em nível lexical com a interação observada entre N e NF.

Uma possibilidade de se fazer isso é levar em consideração a distinção entre vizinhos ortográficos que são compartilhados e vizinhos ortográficos que não são (Perry et al., 2008). Por exemplo, a palavra 'picos' e seu vizinho ortográfico de maior frequência de ocorrência - a palavra 'ricos' - têm, em comum, os vizinhos ortográficos 'bicos' e 'micos', enquanto as palavras 'pinos' e 'pisos' são vizinhos ortográficos apenas da palavra 'picos'. Com base nessa distinção, Perry et al. (2008) predisseram que, em estudos com a técnica de priming, a palavra alvo poderia ser inibida pelos vizinhos ortográficos que ela compartilha com o prime. Assim sendo, seguindo esse raciocínio, se a palavra 'bola' fosse precedida pelo prime ' $\mathrm{b} \# \mathrm{la}$ ', ela tenderia a sofrer inibição das palavras 'bala', 'bela' e 'bula', porque o prime pré-ativaria essas palavras juntamente com a palavra 'bola'. Ora, em uma tarefa que não envolve priming, pode-se argumentar que os vizinhos ortográficos não compartilhados é que passam a ter um papel importante. Isso ocorreria porque os vizinhos ortográficos da palavra alvo que não são compartilhados com o vizinho de maior frequência de ocorrência seriam inibidos apenas pela palavra alvo, só que, nesse caso, como a palavra alvo está também recebendo inibição de seu vizinho ortográfico mais frequente, essa não conseguiria inibir esses vizinhos tão rapidamente quanto uma palavra sem vizinhos ortográficos mais frequentes faria e, dessa forma, eles teriam mais possibilidade de interferir no processo de identificação lexical, explicando, assim, porque o efeito de NF foi mais forte nesse estudo quando as palavras tinham muitos vizinhos ortográficos. Em suma, enquanto a palavra alvo e seu vizinho ortográfico de maior frequência competem acirradamente, os vizinhos da palavra alvo que essas duas unidades lexicais não compartilham têm tempo de aumentar seus níveis de ativação e se tornarem competidores mais fortes, retardando o reconhecimento da palavra alvo e amplificando o efeito inibidor do vizinho ortográfico de maior frequência de ocorrência. No caso dos vizinhos ortográficos compartilhados, a interferência seria menor porque esses seriam inibidos por duas fontes (a palavra alvo e seu vizinho ortográfico de maior frequência) e, se forem considerados os vizinhos ortográficos das palavras que não têm vizinhos ortográficos mais frequentes, pode-se dizer que esses também não causariam tanta interferência porque, embora tenham como fonte de inibição apenas a palavra alvo, essa fonte seria relativamente forte já que ela não sofre com a competição de um vizinho ortográfico mais frequente.

Pode-se dizer que os estudos mais comparáveis ao atual, são os de Justi e Pinheiro (2006, 2008) porque foram desenvolvidos com falantes do português brasileiro e também utilizaram a tarefa de decisão lexical do tipo $G o /$ NoGo. Assim sendo, ao se comparar os resultados desses estudos com os do presente estudo, pode-se dizer que eles são bastante compatíveis, uma vez que os efeitos obser- 
vados foram todos de natureza inibidora. No entanto, nos estudos de Justi e Pinheiro (2006, 2008), tanto N quanto $\mathrm{NF}$ apresentaram efeitos inibidores estatisticamente significantes e, no presente estudo, isso ocorreu apenas com NF. Porém, o fato de efeitos inibidores de $\mathrm{N}$ e NF terem sido estatisticamente significantes nos estudos de Justi e Pinheiro $(2006,2008)$ não é muito surpreendente, já que, em tarefas de decisão lexical a produção dos fonemas necessários à pronúncia dos estímulos não é um processo obrigatório (o que torna o processo de acesso lexical ainda mais saliente).

\section{Experimento B - Tarefa de Leitura em Voz Alta}

O objetivo desse experimento é observar os efeitos de $\mathrm{N}$ e NF quando o processo de conversão grafema/fonema é enfatizado. Para tal, utilizou-se uma tarefa de leitura na qual palavras e pseudopalavras foram apresentadas em uma mesma sessão, devendo o participante ler em voz alta ambos os tipos de estímulo. Nessas situações é comum os participantes lerem todos os estímulos utilizando um processo de conversão grafema/fonema, sem, necessariamente, acessar a representação lexical específica da palavra alvo Coltheart (1978).

\section{Material}

Nesse experimento foram utilizadas as mesmas 64 palavras empregadas previamente no 'Experimento A'. No entanto, para compor a tarefa de leitura em voz alta desse experimento, foram selecionadas 16 novas pseudopalavras dissílabas com muitos vizinhos ortográficos (média de 6,06 e desvio-padrão de 2,94 vizinhos ortográficos).

\section{Procedimentos}

O mesmo esquema de contrabalanceamento de listas que foi empregado no experimento anterior com as 64 palavras correspondentes à manipulação fatorial de $\mathrm{N} \mathrm{e}$ NF também foi empregado nesse experimento. Porém, tomou-se o cuidado de que cada participante fosse exposto a 16 palavras diferentes das que tinha sido exposto previamente no 'Experimento A'. Desse modo, na sessão experimental, cada participante foi exposto a 32 estímulos, sendo 16 palavras e 16 pseudopalavras.

Os procedimentos e instrumentos de testagem foram os mesmos utilizados no 'Experimento A', sendo diferentes, nesse caso, apenas as instruções e a necessidade de se responder a ambos os estímulos. Assim sendo, as instruções foram as seguintes:

Nesse teste você verá esse sinal + no centro da tela. Logo após, você verá uma palavra real ou uma palavra inventada. Você deve ler em voz alta ambos os tipos de estímulos. Tente fazer isso o mais rápido possível, mas evite errar. Aperte o botão 3 para iniciar o treinamento. Os estímulos foram apresentados na mesma fonte e configuração de tela do experimento anterior e com a mesma dinâmica de apresentação, sendo a única diferença a necessidade de se ler em voz alta as palavras e as pseudopalavras. Tal como no experimento anterior, o presente experimento também empregou uma sessão de treinamento que contou com um feedback quanto à precisão das respostas dadas. Caso o participante apresentasse uma porcentagem de erros maior do que $15 \%$ na sessão de treinamento, essa era automaticamente repetida. O tempo total de testagem, incluindo as sessões de treinamento e experimental, foi de, aproximadamente, 5 minutos por participante.

\section{Resultados}

Tal como no 'Experimento A', o método das medidas semirrestringidas (Perea, 1999) foi aplicado ao TR, sendo alterados $4,2 \%$ do total de escores desse experimento, resultando em uma distribuição normal do TR (Kolmogorov-Smirnov $Z=0,494 ; p>0,96)$. O TR médio foi de $773,27 \mathrm{~ms}$, com um desvio padrão de $120,20 \mathrm{~ms}$. Como as pseudopalavras serviram apenas para compor a sessão experimental, a Tabela 2 considera apenas as respostas às palavras e apresenta as estatísticas descritivas para o TR médio dos participantes por condição experimental.

Tabela 2

Média e Desvio Padrão do TR na Tarefa de Leitura em Voz Alta

\begin{tabular}{ccccc}
\hline & \multicolumn{2}{c}{ N1 } & NF1 & NF2 \\
\cline { 2 - 5 } & NF1 & NF2 & 747,03 & 737,19 \\
Média & 759,59 & 756,44 & 118,61 & 110,64 \\
Desvio Padrão & 125,71 & 119,94 & &
\end{tabular}

Nota. N1 = apenas 1 vizinho ortográfico; $\mathrm{N} 2=4$ ou mais vizinhos ortográficos; NF1 = sem vizinhos ortográficos de maior frequência de ocorrência; NF2 = um vizinho ortográfico de maior frequência de ocorrência.

Uma análise de variância considerando N e NF como fatores intra-sujeitos com dois níveis cada e considerando o esquema de contrabalanceamento de listas a partir de dois fatores entre-sujeitos foi desenvolvida da mesma forma que no 'Experimento A'. Além disso, também foram utilizadas, nesse experimento, análises unicaudais para avaliar os efeitos principais de N e NF, já que a partir da proposta de Perea e Rosa (2000) pode-se prever um efeito facilitador de N e NF nessa tarefa. Destarte, considerando-se o efeito principal de $\mathrm{N}$, as palavras com quatro ou mais vizinhos ortográficos foram reconhecidas mais rapidamente (média de $742,11 \mathrm{~ms}$ ) do que as palavras 
Justi, F. R. R., Roazzi, A. \& Justi, C. N. G. (2013). Leitura em Voz Alta e Vizinhança Ortográfica: Acesso Lexical e Processamento Estratégico.

com apenas um vizinho ortográfico (média de $758,01 \mathrm{~ms}$ ), podendo esse efeito ser considerado significante $[F(1,30)$ $=2,711 ; p=0,055$ unicaudal]. As palavras com um vizinho ortográfico de maior frequência de ocorrência também foram reconhecidas mais rapidamente (média de 746,81 ms) do que as palavras sem vizinhos de maior frequência de ocorrência (média de $753,31 \mathrm{~ms}$ ), porém, o efeito principal de NF não foi estatisticamente significante $[F(1,30)=$ 1,$085 ; p=0,15$ unicaudal]. Além disso, a interação entre $\mathrm{N}$ e NF também não foi estatisticamente significante $[F$ $(1,30)=0,18 ; p=0,67]$. Por fim, os participantes apresentaram uma média de porcentagem de erros ínfima $(0,39 \%)$ e, por conseguinte, os dados referentes a essa medida não foram analisados.

\section{Discussão}

De uma forma geral, pode-se dizer que os resultados foram consistentes com as hipóteses experimentais, já que os efeitos de $\mathrm{N}$ e NF apresentaram a direção prevista e o efeito facilitador de $\mathrm{N}$ (aproximadamente, $16 \mathrm{~ms}$ ) foi numericamente maior do que o efeito facilitador de NF (aproximadamente, 6ms). Pode-se argumentar, também, que o fato de apenas o efeito principal de $\mathrm{N}$ poder ser considerado significante, não é especialmente problemático para a proposta de Perea e Rosa (2000), já que esses autores ressaltam que em situações onde o importante é decodificar o estímulo visual em um conjunto de letras, o número de vizinhos ortográficos é mais importante do que a frequência de ocorrência desses vizinhos. Isso ocorre porque esses autores interpretam o efeito de $\mathrm{N}$ como sendo proveniente da retroalimentação que as representações lexicais das palavras enviam para as representações das letras que as compõem (como o feedback que ocorre do nível da palavra para o nível da letra nos modelos de Coltheart et al., 2001; Grainger \& Jacobs, 1996 e McClleland, \& Rumelhart, 1981). Esse processo seria mais sensível ao número de vizinhos ortográficos porque quando uma letra ativa uma palavra, essa palavra retroalimenta suas letras componentes e, assim sendo, um conjunto de letras que ativa muitas palavras recebe de volta mais ativação. Uma explicação complementar para esse efeito facilitador de N é argumentar que, durante a leitura, os vizinhos ortográficos também pré-ativariam os fonemas que compartilham com a palavra alvo, facilitando, assim, o processo de conversão grafema-fonema (Adelman \& Brown, 2007; Mulatti et al., 2006). Isso explicaria porque tem sido observado um efeito facilitador de $\mathrm{N}$ nas tarefas de leitura em voz alta em diferentes línguas alfabéticas como, por exemplo, o inglês (Andrews, 1989), o espanhol (Carreiras et al., 1997) e o francês (Peereman \& Content, 1995). Afinal, nessas tarefas, o efeito de $\mathrm{N}$ seria proveniente de duas fontes: a retroalimentação que as representações lexicais das palavras enviam para as letras que as compõem e a pré-ativação de fonemas compartilhados entre a palavra alvo e seus vizinhos ortográficos.

\section{Conclusão}

Comparando-se os resultados dos dois experimentos realizados, pode-se argumentar que os efeitos de N e NF variam de acordo com as demandas das tarefas ao processo de acesso lexical. Na tarefa de 'Leitura em Voz Alta do Tipo Go/NoGo', onde há uma ênfase no processo de acesso lexical, NF apresentou um efeito inibidor quando as palavras tinham muitos vizinhos ortográficos e na tarefa de 'Leitura em Voz Alta', que visou enfatizar o processo de conversão grafema fonema, $\mathrm{N}$ apresentou um efeito facilitador. O fato dos efeitos de $\mathrm{N}$ e NF poderem ser modulados pela ênfase das tarefas no processo de acesso lexical é compatível com o resultado de outros estudos que investigaram essa questão em versões diferentes da tarefa de decisão lexical (Carreiras et al., 1997; Grainger \& Jacobs, 1996; Justi \& Pinheiro, 2006) e se coaduna com a proposta de Perea e Rosa (2000) de que o efeito dessas variáveis deve-se a processos que ocorrem em momentos diferentes (um processo inicial de retroalimentação no caso de $\mathrm{N}$ e um processo posterior de inibição lateral em nível lexical no caso de NF).

É importante ressaltar que, até onde se sabe, esse é o primeiro trabalho a demonstrar que os efeitos de $\mathrm{N}$ e NF podem ser modulados pela variação na ênfase dada ao processo de acesso lexical em tarefas de leitura em voz alta, sendo, inclusive, o primeiro a investigar o efeito de $\mathrm{N}$ e NF em uma tarefa de leitura em voz alta do tipo Go/ NoGo e o primeiro a investigar o efeito dessas variáveis em tarefas de leitura em voz alta realizadas com falantes do português brasileiro. Pode-se dizer que os resultados desse estudo, tomados em conjunto com os de Justi e Pinheiro (2006, 2008), demonstram que, pelo menos no português brasileiro, quando o acesso lexical é enfatizado o efeito de NF é inibidor e que quando processos extra-lexicais estão mais envolvidos o efeito de $\mathrm{N}$ é facilitador.

Por fim, embora o presente estudo tenha corroborado a proposta de Perea e Rosa (2000) ao observar um efeito inibidor de NF na tarefa de leitura em voz alta do tipo $G o /$ NoGo e um efeito facilitador de $\mathrm{N}$ na tarefa de leitura em voz alta, é importante que estudos futuros realizados com falantes de outras línguas tentem replicar esses achados utilizando essas mesmas tarefas. Outra questão que merece mais investigações é o papel do número de vizinhos ortográficos que não são compartilhados pela palavra alvo e seu vizinho ortográfico de maior frequência de ocorrência. Afinal, considerações acerca dessa nova variável foram importantes para explicar porque o efeito inibidor de NF no 'Experimento $A$ ' foi restrito às palavras que tinham muitos vizinhos ortográficos.

\section{Referências}

Adelman, J., \& Brown, G. (2007). Phonographic neighbors, not orthographic neighbors, determine word naming latencies. Psychonomic Bulletin \& Review, 14, 455-459. 
Andrews, S. (1989). Frequency and neighborhood effects on lexical access: Activation or search? Journal of Experimental Psychology: Learning, Memory \& Cognition, 15, 802-814.

Andrews, S. (1997). The effect of orthographic similarity on lexical retrieval: Resolving neighborhood conflicts. Psychonomic Bulletin \& Review, 4, 439-461.

Carreiras, M., Perea, M., \& Grainger, J. (1997). Effects of orthographic neighborhood in visual word recognition: Cross-task comparisons. Journal of Experimental Psychology: Learning, Memory, \& Cognition, 23, 857-871.

Clark, H. (1973). The language-as-fixed-effect fallacy: A critique of language statistics in psychological research. Journal of Verbal Learning and Verbal Behavior, 12, 335-359.

Coltheart, M. (1978). Lexical access in simple reading tasks. In G. Underwood (Ed.), Strategies of information processing (pp. 151-216). New York: Academic Press.

Coltheart, M., Davelaar, E., Jonasson, J., \& Besner, D. (1977). Access to the internal lexicon. In S. Dornic (Ed.), Attention and performance VI (pp. 535-555). Hillsdale, NJ: Erlbaum.

Coltheart, M., Rastle, K., Perry, C., Langdon, R., \& Ziegler, J. (2001). DRC: A dual route cascaded model of visual word recognition and reading aloud. Psychological Review, 108, 204-256.

Forster, K., \& Forster, J. (2003). DMDX: A windows display program with millisecond accuracy. Behavior Research Methods, Instruments and Computers, 35, 116-124.

Grainger, J., \& Jacobs, A. (1996). Orthographic processing in visual word recognition: A multiple read-out model. Psychological Review, 103, 518-565.

Grainger, J., O’Reagan, K., Jacobs, A., \& Segui, J. (1989). On the role of competing word units in visual word recognition: The neighborhood frequency effect. Perception \& Psychophysics, 45, 189-195.

Huntsman, L., \& Lima, S. (2002). Orthographic neighbors and visual word recognition. Journal of Psycholinguistic Research, 31, 289-306.

Justi, F. R. R., \& Justi, C. N. G. (2008). As estatísticas de vizinhança ortográfica do português e do inglês são diferentes? Psicologia em Pesquisa, 2, 61-73.

Justi, F. R. R., \& Pinheiro, A. M. V. (2006). O efeito de vizinhança ortográfica no português do Brasil: Acesso lexical ou processamento estratégico. Interamerican Journal of Psychology, 40, 275-288.

Justi, F. R. R., \& Pinheiro, A. M. V. (2008). O efeito de vizinhança ortográfica em crianças brasileiras: Estudo com a tarefa de decisão lexical. Interamerican Journal of Psychology, 42, 559-569.

Mathey, S. (2001). L'influence du voisinage orthographique lors de la reconnaissance des mots écrits. Revue Canadienne de Psychologie Expérimentale, 55, 1-23.

McClleland, J., \& Rumelhart, D. (1981). An interactive activation model of context effects in letter perception: Pt. 1, an account of basic findings. Psychological Review, 88, 375-407.

Monsell, S., Doyle, M., \& Haggard, P. (1989). Effects of frequency on visual word recognition tasks: Where are they? Journal of Experimental Psychology: General, 118, 43-71.

Mulatti, C., Reynolds, M., \& Besner, D. (2006). Neighborhood effects in reading aloud: New findings and new challenges for computational models. Journal of Experimental Psychology: Human Perception and Performance, 32, 799-810.

Peereman, R., \& Content, A. (1995). Neighborhood size effect in naming: Lexical activation or sublexical correspondences? Journal of Experimental Psychology: Learning, Memory, \& Cognition, 21, 409-421.
Perea, M. (1999). Tiempos de reacción y psicología cognitiva: dos procedimientos para evitar el sesgo debido al tamaño muestral. Psicológica, 20, 13-21.

Perea, M., \& Pollatsek, A. (1998). The effects of neighborhood frequency in reading and lexical decision. Journal of Experimental Psychology: Human Perception \& Performance, 24,767-779.

Perea, M., \& Rosa, E. (2000). The effects of orthographic neighborhood in reading and laboratory word identification tasks: A review. Psicológica, 21, 327-340.

Perry, J., Lupker, S., \& Davis, C. (2008). An evaluation of the interactive-activation model using masked partial-word priming. Language and Cognitive Processes, 23, 36-68.

Pinheiro, A. M. V., \& Reis, C. (2004). A influência da natureza fonética do fonema inicial da palavra na medida de tempo de reação em tarefas de leitura em voz alta. Revista de Estudos da Linguagem, 12, 19-36.

Pollatsek, A., \& Well, A. D. (1995). On the use of counterbalanced designs in cognitive research: A suggestion for a better and more powerful analysis. Journal of Experimental Psychology: Learning, Memory, and Cognition, 21, 785-794.

Raaijmakers, J., Schrijnemakers, J., \& Gremmen, F. (1999). How to deal with "the language-as-fixed-effect fallacy": Common misconceptions and alternative solutions. Journal of Memory and Language, 41, 416-426.

Siakaluk, P., Sears, C., \& Lupker, S. (2002). Orthographic neighborhood effects in lexical decision: The effects of nonword orthographic neighborhood size. Journal of Experimental Psychology: Human Perception and Performance, 28, 661-681.

Treiman, R., Mullennix, J., Bijeljac-Babic, R., \& RichmondWelty, E. (1995). The special role of rimes in the description, use, and acquisition of English orthography. Journal of Experimental Psychology: General, 124, 107-136.

Warner, R. M. (2008) Applied statistics: From bivariate through multivariate techniques. London: Sage.

Ziegler, J., \& Perry, C. (1998). No more problems in Coltheart's neighborhood: Resolving neighborhood conflicts in the lexical decision task. Cognition, 68, B53-B62. 


\section{Anexo}

Palavras Correspondentes à Manipulação Fatorial de N e NF Utilizadas nas Tarefas de Leitura em Voz Alta do Experimento A e do Experimento B

\begin{tabular}{|c|c|c|c|c|}
\hline & \multicolumn{2}{|c|}{$\mathrm{N}=1$ vizinho ortográfico } & \multicolumn{2}{|c|}{$\mathrm{N} \geq 4$ vizinhos ortográficos } \\
\hline & $\mathrm{NF}=0$ vizinhos & $\mathrm{NF}=1$ vizinho & $\mathrm{NF}=0$ vizinhos & $\mathrm{NF}=1$ vizinho \\
\hline \multirow[t]{4}{*}{ Lista 1} & ASTRO & ONZE * & ASAS & ÁSIA \\
\hline & BISPO & BALCÃO & BURRO & BARES \\
\hline & DUPLAS & DIVÃ & DICAS & DATAS \\
\hline & FAUNA & FICHAS & FERAS & FADAS \\
\hline \multirow[t]{4}{*}{ Lista 2} & BICHOS & BONÉ & BRIGAS & BARRAS \\
\hline & CRENÇA & CRISES & CALMA & CABOS \\
\hline & SAQUE & SABOR & SUCO & SACO \\
\hline & TRUQUE & TRIBO & TAMPA & TRAVE \\
\hline \multirow[t]{4}{*}{ Lista 3} & CAPIM & CRIVO & CRAVO & CABRA \\
\hline & FILTRO & FUSCA & FARDOS & FUNDA \\
\hline & MANHÃS & MUNDOS & MAPAS & MUROS \\
\hline & PADRES & PATRÃO & PINGA & PICOS \\
\hline \multirow[t]{4}{*}{ Lista 4} & CACAU & CARNÊS & CASCA & CALDO \\
\hline & CREDOR & CORAIS & CONDE & CALÇA \\
\hline & FIBRAS & FRANGO & FITAS & FUMO \\
\hline & TAMPÃO & TRONO & TUMBA & TOMBO \\
\hline
\end{tabular}

Nota. * O fonema inicial /õ/ foi pareado com o fonema inicial /a/ das palavras 'ASTRO', 'ASAS' e 'ÁSIA', porque Pinheiro e Reis (2004) não observaram diferenças estatisticamente significativas entre as médias de tempo de reação das palavras iniciadas com esses fonemas $(/ \tilde{\mathrm{o}} / \mathrm{e} / \mathrm{a} /)$. 